\title{
The Reliability of the Anaerobic Power Dummy Throw Test in High School Wrestlers: A Pilot Study
}

\author{
Eric Lopez ${ }^{1}$, Ryan Luke ${ }^{2}$, Kent J. Adams ${ }^{2}$ and Mark DeBeliso ${ }^{1}$ \\ 1. Department of Kinesiology and Outdoor Recreation, Southern Utah University, Cedar City, UT 84720, USA \\ 2. Department of Kinesiology, California State University Monterey Bay, Seaside, CA 93955, USA
}

\begin{abstract}
AP (anaerobic power) output is an important physical characteristic that is required to succeed in sports such as wrestling. The Wingate test is considered the Gold Standard for assessing AP but is not specific to the sport of wrestling. The purpose of this study was to examine the reliability of a novel field test known as the LWAPT (Lopez wrestling anaerobic power test) for the assessment of AP. The participants were male high school wrestlers $(n=10$, age: $17.0 \pm 0.8 \mathrm{yrs}$, mass: $70.9 \pm 10.2 \mathrm{kgs})$. The participants met on one occasion in order to complete the testing protocol. The protocol initiated with the participants completing a 10-15 minute dynamic WU (warm-up) that included three practice dummy throw attempts (dummy mass $=31.75 \mathrm{kgs}$ ). Following the dynamic WU $(\approx 5 \mathrm{minutes})$, the participants performed two trials of the LWAPT. In order to perform the LWAPT, wrestlers stood behind the wrestling dummy in a squat position with legs bent at 45-90 degrees. Next the wrestlers wrapped their arms around the waist of the dummy and on the signal "go" the wrestlers quickly exploded up, lifting the dummy by getting triple extension with the ankles, knees and hips as one would in a power clean. After the wrestler was fully extended, he turned in midair in order to drive the dummy onto its stomach on the ground, where the wrestler was on top of the back of the dummy (a common position after an opponent has been thrown). The participant then repositioned the dummy to the original position to execute another throw. The participants completed as many dummy throws as possible during the one minute trials. The LWAPT trials were separated by 15 minutes. The trial scores were $15.6 \pm 2.5$ and $17.2 \pm 1.5$ throws respectively. The interclass and intraclass reliability coefficients were $r=0.84$ and ICC $=0.80$. The standard error of the measure was $\mathrm{SEm}_{\mathrm{m}}=1.0$ throws with $90 \%$ confidence limits of $\mathrm{U}_{\mathrm{L}}: 1.7, \mathrm{~L}_{\mathrm{L}}: 0.7$. The mean difference between trials was $1.6 \pm 1.4$ throws (90\% confidence limits of $\mathrm{UL}_{\mathrm{L}}: 2.4$, LL: 0.8). Bland-Altman plots suggested agreement between trials with no evidence of heteroscedasticity. The LWAPT exhibits moderate to high reliability as an assessment of AP. The inclusion of additional dummy throw trials to the assessment protocol may enhance the degree of reliability of the dummy throw test as a measure of AP.
\end{abstract}

Key words: Reliability, dummy, wrestling, high school.

\section{Introduction}

Wrestling is considered the oldest sport in history with evidence from as far back as 5,000 years, which includes artifacts that had illustrations of wrestlers on them [1]. Pindar the Greek poet describes the battle between the gods Zeus and Cronus as a wrestling match for control of the universe in which Zeus was triumphant, and Olympic festivals dating back to the Eighth Century B.C. honored his victory [1]. From ancient times to this day, wrestling has been a popular sport.

Corresponding author: Mark DeBeliso, $\mathrm{PhD}$, Professor, research fields: orthopedic biomechanics, mechanics and metabolics of sport movements and work-tasks, strength training, and master's athletes.
The National Wresting Coaches Association [2] reports significant increase in participation in all levels - from high school to Olympic - for both men's and women's wrestling since the mid 1990's. Participation in U.S. high school women's wrestling has increased $1,814 \%$, from 804 wrestlers in 1994 to 14,587 in 2016 . There has been more than 180 new or reinstated collegiate wrestling teams established since 2001 and women's wrestling became part of the Olympics in 2004 [2]. Not only has participation in wrestling shown a dramatic increase in the last 30 years, but wrestling has also become an integral part of another widely popular sport, MMA (mixed martial arts), which incorporates specific grappling techniques unique to wrestling. For being such a popular sport, 
very little research and no field tests exist for wrestling that require physical prowess.

The sport of wrestling relies on maximal strength, balance, agility, and isometric force during whole body movements, and AP (anaerobic power). AP is an important physical characteristic that is required to succeed in sports such as wrestling because one must be able to create continuous explosive movements for up to a total of six minutes with very little rest. The primary energy systems that provide the fuel are the adenosine triphosphate phosphocreatine (ATP-PC), which provides 5-15 seconds of energy, and glycogen (anaerobic glycolysis), which provides 1-3 minutes of energy [3]. Lansky et al. [4] analyzed wrestling matches and calculated during a two-minute round, an explosive attack was performed around every 6-10 seconds. The ability to create explosive movements over time has become known as power-endurance [5, 6]. Conchola et al. [5] states that power endurance is a major characteristic in sports which require explosiveness. Yoon et al. [6] describes this characteristic as "muscular endurance in which it is the ability to sustain muscular performance at a high intensity, that is at or near $100 \%$ of maximum force or power for more than 30 seconds, but less than two minutes" [6]. Training for power-endurance can be very demanding; however, the athlete that possesses the greatest amount of power-endurance may have a better chance of winning the match.

Research establishing field tests for the purpose of assessing physical preparedness to compete in wrestling fail to fully address the specific movement patterns engaged in during wrestling [7-10] or have other limitations [9]. In other sports that have not been around as long as wrestling, field tests have been researched and validated. For example, the sport of North American football has been around for a little over a hundred years but has validated field tests such as the 40-yard dash, pro agility drill, and broad jump to name a few [11-13]. These field tests can be conducted almost anywhere in open space, are cost effective and do not need professional expertise.

One field test that exists that shows reliability for physiological readiness for the sport of wrestling is the sandbag throw test devised by Wright et al. [10]. This test consists of a repeated series of lifting, throwing from a stationary position to a shoulder-height line on a wall, and then dragging a sandbag [10]. A benefit of this test is that when athletes lift the sandbag, they get triple extension like they would in a power clean. The drawbacks of this test, however, are that the motions required in this test do not simulate the physical motions required of a wrestler (i.e. lack specificity). The sandbag test involves a forward thrusting motion that results in the object being thrown against a wall, wrestlers use more of a controlled, push-pull motion that keeps the opponent within arm-distance. In addition, a wrestler does not use the motion of dragging the object backward in order to throw it repeatedly.

The wrestling dummy is a commonly used training tool that allows a wrestler to practice throwing in a manner that is very similar to throwing an actual opponent (i.e. specificity). The wrestling dummy comes in a wide range of weights and heights. Wrestlers can practice their moves on the dummy as well as lifting and throwing it as one would in a wrestling match. As such, developing a field test utilizing the wrestling dummy to test the physical capacity required to engage at a high level in wrestling warrants investigation. If a field test using the wrestling dummy could be developed to assess AP (with acceptable reliability) then coaches and athletes would have a cost effective means for assessing AP in a wrestling sport specific manner.

Hence, the purpose of this study was to explore the reliability of a novel field test known as the LWAPT (Lopez wrestling anaerobic power test) for the assessment of AP. 


\section{Methods}

\subsection{Participants}

The participants for this study were high school male wrestlers from Gilroy (California, US) high school who were recruited and later volunteered for the study. Permission to engage the study was obtained through a University Institutional Review Board before any testing was conducted. The University Institutional Review Board provides ethical oversight for the protection of rights of human subjects in research studies. Each participant was given an Institutional Review Board approved written consent form that was signed by each participant and their parent prior to engaging in the study. The study occurred during a break in the season when no weight restriction was taking place.

\subsection{Procedures}

The participants met on one occasion in order to complete the testing protocol. The testing session took place in the wrestling room at Gilroy high school. The participants had no tournaments or competition for two weeks prior to data collection. As such, there was no weight cutting or dieting prior to testing. The testing procedure began with the participants completing a 10-15 minute dynamic WU that included three practice dummy throw attempts. The dynamic WU included jogging, tumbling forward rolls, cart wheels, duck walks, shadow wrestling, tie-ups, finishing shots, and sprints.

Following the dynamic WU, the participants performed two trials of the LWAPT. In order to perform the LWAPT, wrestlers stood behind the wrestling dummy in a squat position with legs bent at 45-90 degrees (Fig. 1). Next the wrestlers wrapped their arms around the waist of the dummy and on the signal "go" the wrestlers quickly exploded up, lifting the dummy by getting triple extension with the ankles, knees and hips as when performing a power clean. After the wrestler was fully extended, he turned in midair in order to drive the dummy onto its stomach on the ground, where the wrestler was on top of the back of the dummy (a common position after an opponent has been thrown).

The participant then repositioned the dummy to the original position to execute another throw. The participants completed as many dummy throws as possible during the one minute trials. The LWAPT trials were separated by 15 minutes. The mass of the dummy was $31.75 \mathrm{kgs}$.

\subsection{Statistical Analysis}

Multiple statistical approaches were used to quantify the test-retest reliability of the LWAPT (trial 1 vs. trial 2). Interclass ( $r$ ) and intraclass coefficients (ICC) of reliability were calculated as well as the standard error of the measure $\left(\mathrm{SE}_{\mathrm{m}}\right)$. Scatter diagram and Bland-Altman plots were constructed to examine linearity and uniformity of error. The data were also

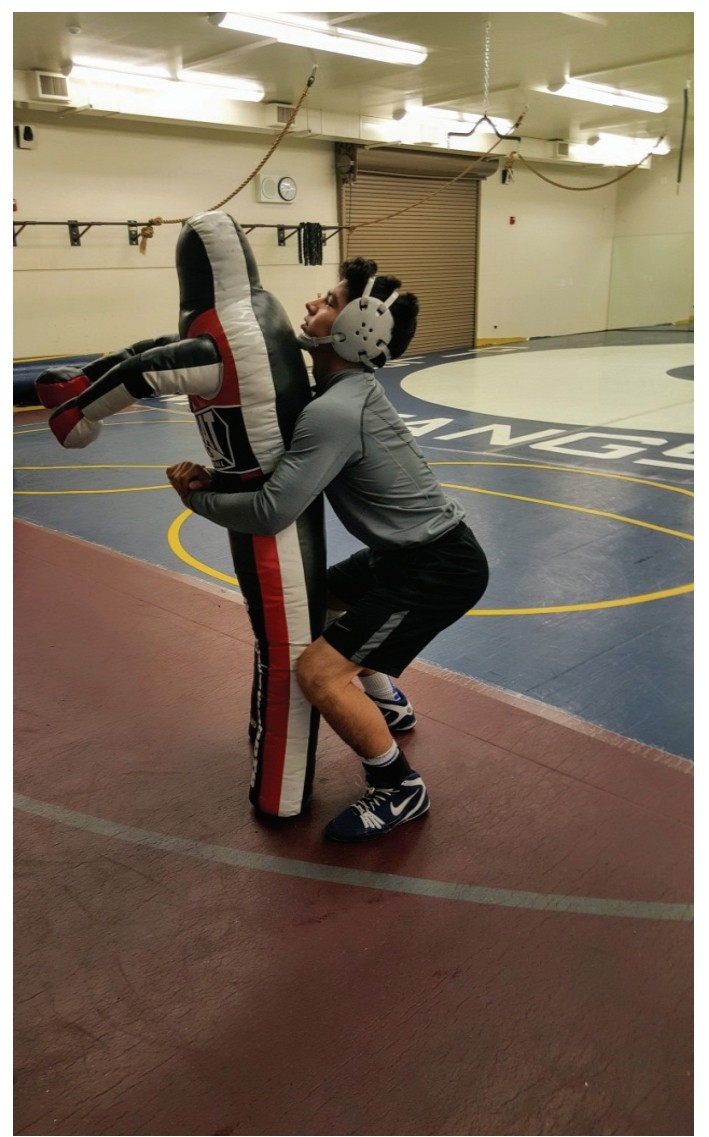

Fig. 1 Starting position for dummy throw (mass $31.75 \mathrm{kgs}$ ). 
Log-transformed in order to assess typical error in the form of a coefficient of variation percent (CV\%). Ninety percent confidence limits were also calculated for the reliability statistics. Excel was used for statistical analysis with a spreadsheet developed by Will Hopkins [14]. This multiple approach reliability analysis is consistent with several previously reported [15-19].

\section{Results}

Ten high school male wrestlers participated in the study. The age, height, and body mass of the participants are provided in Table 1. All of the participants completed both test trials of the LWAPT without complication.

The reliability analysis is based on one session of test re-test trial scores of the LWAPT $(n=10)$. The mean and standard deviation of trial 1 and trial 2 scores from both trials were $15.6 \pm 2.5$ and $17.2 \pm 1.5$ throws respectively, with an average difference between trials of $1.6 \pm 1.4$ throws. Fig. 2 is a scatter plot of trial 1 and trial 2 of dummy throw scores. The scatter plot suggests a linear relationship between the LWAPT trial scores. Fig. 3 is a Bland-Altman plot comparing trial LWAPT average scores versus the difference scores. The Bland-Altman plot demonstrates that none of the difference scores exceeded the $95 \%$ limits of agreement, considered reliable [20]. Neither the Bland-Altman plot nor the scatter plot provided evidence of bias or heteroscedasticity (non-uniform error). Of the 10 pairs of LWAPT scores, one individual scored higher on the first trial, one scored the same on both trials, and 8 scored higher during trial 2.

Table 2 provides the reliability indicators with $90 \%$ confidence limits $\left(\mathrm{U}_{\mathrm{L}}, \mathrm{L}_{\mathrm{L}}\right)$. The intraclass coefficient was ICC $=0.80(0.93,0.49)$, which is considered average acceptable [21]. The interclass reliability coefficient was $r=0.84(0.95,0.54)$, which is considered high [22]. The standard error of the measure was $\mathrm{SE}_{\mathrm{m}}=1.0(1.7,0.7)$ throws. While the trial data did not suggest non-uniform error, we decided to calculate typical error (CV\%), as such the data were Log-transformed as described by Hopkins [23]. The typical error or coefficient of variation was $\mathrm{CV} \%=7.6$ $(13.3,5.4)$ percent.

Table 1 Participant descriptive data.

\begin{tabular}{lll}
\hline Age (years) & Height $(\mathrm{cm})$ & Mass $(\mathrm{kg})$ \\
\hline $17.0 \pm 0.8$ & $173.3 \pm 6.7$ & $70.9 \pm 10.2$ \\
\hline
\end{tabular}

High school male wrestlers, $\mathrm{N}=10$ (mean $\pm \mathrm{sd})$.

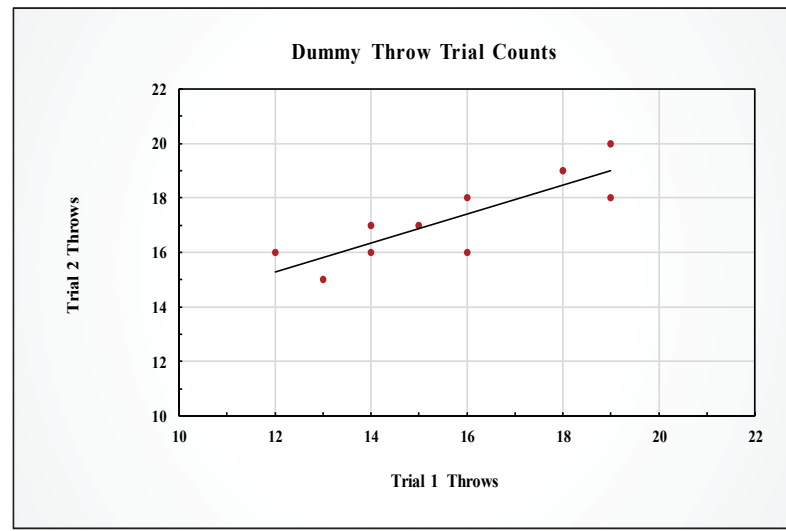

Fig. 2 Scatter plot of dummy throw scores.

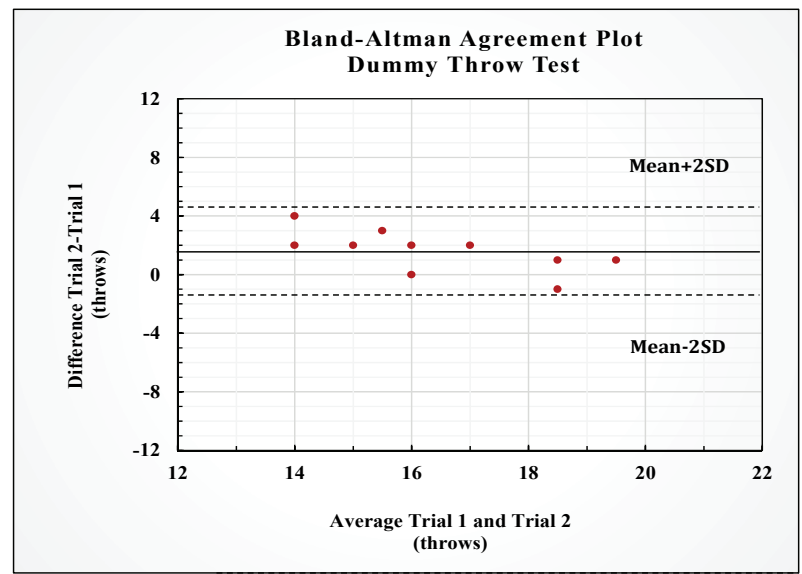

Fig. 3 Bland- Altman plot of dummy throw trial scores.

Table 2 Dummy throw reliability statistics.

\begin{tabular}{llcc}
\hline Statistic & & Upper limit & Lower limit \\
\hline$\Delta$ Means (throws) & $1.6 \pm 1.4$ & 2.4 & 0.8 \\
$r$ & 0.84 & 0.95 & 0.54 \\
ICC & 0.80 & 0.93 & 0.49 \\
Typical error (CV\%)* & 7.6 & 13.3 & 5.4 \\
SEm $_{\mathrm{m}}$ & 1.0 & 1.7 & 0.7 \\
\hline
\end{tabular}

$90 \%$ Confidence limits. Typical error expressed as a CV\% based on Log-transformed data. SEm: Standard error of the measure. $r$ : Pearson correlation. ICC: Intraclass correlation coefficient. 


\section{Discussion}

The purpose of this study was to explore the reliability of a novel field test known as the LWAPT for the assessment of AP. To our knowledge this is the first such data set presented in the literature regarding dummy throw testing. As such, comparison with previous established norms as would be presented in Hoffman [24] is not possible. The data suggest that the LWAPT exhibits moderate to high reliability as an assessment of AP.

The interclass reliability coefficient $(r=0.84)$ is consistent with test-retest reliability coefficients reported for other common physical performance tests [22]. Likewise, the intraclass reliability coefficient (ICC $=0.80)$ is consistent with the ICC's reported for many other commonly used physical performance tests [21].

The standard error of measure $\left(\mathrm{SE}_{\mathrm{m}}\right)$ is a quantification of absolute reliability [25]. The $\mathrm{SE}_{\mathrm{m}}$ in our study suggests that when assessing AP with the LWAPT that the true score is within \pm 1.0 throws of the measured score ( $68 \%$ confidence). The salience here is that if one is using the LWAPT to assess change in AP (increase or decrease), then the score must be greater than one throw of the dummy or it could be that the change in scores observed was measurement error.

Worthy of noting is that when scores exhibit non-uniform error, the $\mathrm{SE}_{\mathrm{m}}$ is biased and typically over-estimates error in the lower scores and under-estimates the error in the higher scores. We did not observe evidence of bias or non-uniformity of error in the data; however we felt it prudent to make a correction to the data to guarantee that the reliability examination was comprehensive.

When data exhibit non-uniformity of error Hopkins [23] recommends log-transforming the scores and expressing the error as typical error (or $\mathrm{CV} \%$ ). The $\mathrm{CV} \%$ represents $(\mathrm{CV} \%=7.6$ percent $)$ a non-bias measure of error when attempting to measure change in LWAPT scores in this population. Worthy of note is that the $\mathrm{CV} \%=7.6$ percent would be 1.2 dummy throws which is nearly equal to the $\mathrm{SE}_{\mathrm{m}}$ in the current study $\left(\mathrm{SE}_{\mathrm{m}}=1.0\right.$ throws). That the $\mathrm{CV} \%$ was nearly equal to the $\mathrm{SE}_{\mathrm{m}}$, is evidence that the data collected were void of bias and/or non-uniformity of error.

In this study, the interclass reliability coefficient was $r=0.84(0.95,0.54)$, which is considered high [22]. In other research, $r \geq 0.80$ is acceptable for physical performance tests [22]. Hence, the interclass reliability coefficient in the current study suggests that the LWAPT exhibits an acceptable level of test-retest reliability.

Most previous research on field tests for wrestlers, such as the sandbag throw conditioning test and the upper-body and lower-body Wingate tests have demonstrated high reliability coefficients ranging from ( $r=0.85$ to 0.97 ) $[7,8,10]$. However, because these tests are not specific to the sport of wrestling and do not replicate the movements wrestlers execute, these results cannot be directly compared to those of the LWAPT. The only documented test specific to wrestling is the PWPT (Pittsburgh wrestling performance test) [9], which explored the validity of the PWPT by comparing it to measures of AP, muscular strength, and the Cleveland State University wrestling performance test [8]. The PWPT has subjects perform repetitions of wrestling-specific moves on human counterparts instead of dummies in order to test for validity of wrestling moves, and the results indicate that this test was both valid and practical for coaches to use with wrestlers without expensive equipment. The researchers state that the primary limitation of this test is the potential influence of slow reaction time on the part of the passive human partner [9], a limitation that using dummies would not be subject to.

AP is widely recognized as predictive of a wrestler's success $[4,6,7,9,10,26-28]$, and the LWAPT both tests for AP and mimics the motions wrestlers make. Between 2 trials, 8 out of 10 subjects were able to score higher on the second trial. This tells us that fatigue was not an issue and/or that the subjects were getting better at the throws. In order to improve the reliability of the 
test, it is suggested to perform a minimum of three trials.

The participants in this study were not familiar with the wrestling dummy and the throwing of it, which likely deterred or lowered the reliability results documented in the current study. Future studies should include participants that are familiar with the wrestling dummy and focus on different populations, such as college or junior high wrestlers, with appropriate alterations in the load of the dummy, in order to determine more precisely the reliability of the LWAPT across a range of the wrestling population. Likewise, additional efforts should focus on additional dummy throw trials in each test session. Length of testing should vary from the junior high level all the way up to the college level as wrestling match periods vary for differing levels of competition.

The dummy mass selected in the current study was approximately 32 kilograms (70 pounds) and was on average $45 \%$ of the wrestler's mass in the current study (32 kilograms/70.1 kilograms). The 32-kilogram dummy used in the current study is inexpensive and easily obtainable at e-commerce sites such Amazon.com. With that said, it is possible that using a dummy mass differing from that used in the current study may improve the reliability of the LWAPT. Specifically, junior high, high school and college wrestlers would likely be better served by training loads based on their age range and physical maturation. Wrestlers at the junior high level should train with a lighter wrestling dummy as well as reducing the duration of the trial to 30 seconds. High school level should train with loads of 32 kilograms (70 lbs.) for the light to middle weight class and the upper weight class should train with a 45-kilogram (or 100 lbs.) dummy. College age wrestlers and above would likely be better served by training with heavier wrestling dummy's and for a longer duration due to their elite maturation and competitive level.

The initial inspiration for the LWAPT came from two drills that legendary college wrestling coach Dan
Gable promotes for conditioning wrestlers. Dan Gable won fifteen national championships while coaching at the University of Iowa from 1976-1997 [29]. The first drill, an over-the-head throw, is not allowed in high school folk-style wrestling but is allowed in Greek and freestyle wrestling. In Gable's drill, the wrestler throws a dummy over his head in a horizontal plane, only extending the hips. The second drill used to develop the LWAPT is a lifting drill, also known as a mat return, in which the wrestler gets triple extension and returns their live partner back to the mat on his side/stomach. The LWAPT modifies both of these drills, using a dummy and adopting the intensity and the hip motion of the throw from the first drill and the explosive movement of the triple extension on the vertical plane from the second drill. The use of the dummy in the LWAPT is to make sure that the subject does not have to worry about potential injury or influence of a live partner but instead can participate in the test without hesitation or distraction.

\section{Conclusions}

Within the parameters of this study, the LWAPT scores demonstrated a moderate to high degree of reliability among high school male wrestlers. The LWAPT is sport specific, economical, and can be executed in virtually any open space, and does not require a high degree of expertise by the wrestler to execute. Further, the LWAPT, when used to assess AP, is an easily administered test. The authors recommend the use of the LWAPT to HS school wrestling coaches and suggest that three trials of the LWAPT will likely yield a reliable sport specific assessment of AP.

\section{References}

[1] Dellinger, B. "The Oldest Sport." Retrieved from http://nwhof.org/stilLWAPTer/resources-library/history/t he-oldest-sport/.

[2] National Wrestling Coaches Association Wrestling's Facts and Resources. 2016. Retrieved from http:/www.nwcaonline.com/growing-wrestling/facts-reso urces/\#scholastic-facts.

[3] Fink, H., and Mikesky, A. 2012. Practical Applications in 
Sports Nutrition. 3rd ed. Sudbury, MA: Jones and Bartlett Publishers.

[4] Lansky, R. 1999. "Wrestling and Olympic-Style Lifts: In-Season Maintenance of Power and Anaerobic Endurance." Journal of Strength and Conditioning Research 21 (3): 21-7.

[5] Conchola, E., and Thiele, R. 2015. "Acute Post Exercise Time Course Responses of Hypertrophic vs. Power-Endurance Squat Exercise Protocols on Maximal and Rapid Torque of the Knee Extensors." Journal of Strength and Conditioning Research 29 (5): 1285-94.

[6] Yoon, J. 2002. "Physiological Profiles of Elite Senior Wrestlers." Sports Medicine 32 (4): 225-33.

[7] Hickner, R. C., and Horswill, C. A. 1991. "Test Development for the Study of Physical Performance in Wrestlers Following Weight Loss." International Journal of Sports Medicine 12 (6): 557-62.

[8] Klinzing, J., and Karpowicz, W. 1983. "A Test to Measure the Performance Capabilities of Wrestlers." Journal of Strength and Conditioning Research 5 (4): 40-3.

[9] Utter, A., Goss, F., and DaSilva, S. 1997. "Development of a Wrestling Specific Performance Test." Journal of Strength and Conditioning Research 11 (2): 88-91.

[10] Wright, G., and Isaacson, M. 2015. "Development and Assessment of Reliability for a Sandbag Throw Conditioning Test for Wrestlers." Journal of Strength and Conditioning Research 29 (2): 451-7.

[11] Sierer, P., and Battaglini, C. 2008. "The National Football League Combine: Performance Differences Between Drafted and Nondrafted Players Entering the 2004 and 2005 Drafts." Journal of Strength and Conditioning Research 22 (1): 6-12.

[12] Bissas, A., and Havenetidis, K. 2008. "The Use of Various Strength-Power Tests as Predictors of Sprint Running Performance." The Journal of Sports Medicine and Physical Fitness 48 (1): 49-54.

[13] Kuzmits, F., and Adams, A. 2008. "The NFL Combine: Does It Predict Performance in the National Football League?" Journal of Strength and Conditioning Research 22 (6): 1721-7.

[14] Hopkins, W. G. 2007. "Understanding Statistics by Using Spreadsheets to Generate and Analyze Samples." Sportscience 11: 23-36.

[15] Ah Sue, R., Sevene, T., Berning, J., Adams, K. J., and DeBeliso, M. 2017. "Determination of Trials Needed for Measurement Consistency of Standing Long Jump in
Female Collegiate Volleyball Athletes.” A Brief Report, International Journal of Sports Science 7 (1): 1-5.

[16] Crandall, K., Phillips, D., and DeBeliso, M. 2017. "The Reliability of Reactive Agility Testing in 5th Grade Females." Journal of Athletic Enhancement 6 (3): 1-4.

[17] Reid, C., Dolan, M., and DeBeliso, M. 2017. "The Reliability of the Standing Long Jump in NCAA Track and Field Athletes." International Journal of Sports Science 7 (6): 233-8.

[18] Tano, G., Bishop, A., Climstein, M., and DeBeliso, M. 2016. "The Reliability of the Prowler in High School Male Football Players.” Journal of Sports Science 4 (4): 183-8.

[19] White, K., DeBeliso, M., Sevene, T., and Adams, K. J. 2015. "The Reliability of the 300-Yard Shuttle Run in High School Girls Basketball Players." Journal of Sports Science 3 (5): 214-8.

[20] Bland, J. M., and Altman, D. G. 1986. "Statistical Methods for Assessing Agreement between Two Methods of Clinical Measurement." Lancet 8: 307-10.

[21] Baumgartner, T. A., Jackson, A. S., Mahar, M. T., and Rowe, D. A. 2007. Measurements for Evaluation in Physical Education and Exercise Science. 8th ed. New York, NY: McGraw Hill.

[22] Safrit, M. J., and Wood, T. M. 1995. Introduction to Measurement in Physical Education and Exercise. 3rd ed. St. Louis, MO: Mosby.

[23] Hopkins, W. G. 2013. "A New View of Statistics." Internet Society for Sport Science, Retrieved from http://www.sportsci.org/resource/stats/.

[24] Hoffman, J. 2006. Norms for Fitness, Performance, and Health. Champaign, IL: Human Kinetics.

[25] Bruton, A., Conway, J. H., and Holgate, S. T. 2000. "Reliability: What Is It and How Is It Measured?" Physiotherapy 86 (2): 94-9.

[26] Horswill, C. A. 1992. "Applied Physiology of Amateur Wrestling." Sports Medicine 14 (2): 114-43.

[27] Horswill, C. A., Scott, J. R., and Galea, P. 1989. "Comparison of Maximum Aerobic Power, Maximum Anaerobic Power, and Skinfold Thickness of Elite and Nonelite Junior Wrestlers." International Journal of Sports Medicine 10 (3): 165-8.

[28] Hubner-Wozniak, E., Kosmol, A., Lutoslawska, G., and Bem, E. Z. 2004. "Anaerobic Performance of Arms and Legs in Male and Female Free Style Wrestlers.” Journal of Science Medicine in Sport 7 (4): 473-80.

[29] Gable, D. 2011. Retrieved from: http://dangable.com/bio/. 